\title{
Oxidative CaMKII as a potential target for inflammatory disease (Review)
}

\author{
JINGJING QU ${ }^{1}$, QUANHUI MEI ${ }^{2}$ and RUICHAO NIU ${ }^{3}$ \\ ${ }^{1}$ Department of Lung Cancer and Gastroenterology, Hunan Cancer Hospital, Affiliated Tumor Hospital \\ of Xiangya Medical School of Central South University, Changsha, Hunan 410008; \\ ${ }^{2}$ Department of Intensive Care Unit, The First People's Hospital of Changde City, \\ Changde, Hunan 410005; ${ }^{3}$ Department of Respiratory Medicine, Xiangya Hospital, \\ Central South University, Changsha, Hunan 410008, P.R. China
}

Received June 20, 2018; Accepted May 22, 2019

DOI: $10.3892 / \mathrm{mmr} .2019 .10309$

\begin{abstract}
CaMKII is a calcium-activated kinase, proved to be modulated by oxidation. Currently, the oxidative activation of CaMKII exists in several models of asthma, chronic rhinosinusitis with nasal polyps, cardiovascular disease, diabetes mellitus, acute ischemic stroke and cancer. Oxidized CaMKII (ox-CaMKII) may be important in several of these diseases. The present review examines the mechanism underlying the oxidative activation of CaMKII and summarizes the current findings associated with the function of ox-CaMKII in inflammatory diseases. Taken together, the findings of this review aim to improve current understanding of the function of ox-CaMKII and provide novel insights for future research.
\end{abstract}

\section{Contents}

1. Introduction

2. CaMKII is activated by ROS

3. Role of ox-CaMKII in inflammatory diseases

4. Conclusions

\section{Introduction}

CaMKII is a multi-polymer serine threonine kinase consisting of 12 subunits. Each subunit contains three conserved domains: i) A catalytic domain in an amino terminal, which supplies the binding site for adenosine triphosphate (ATP) and multiple

Correspondence to: Dr Ruichao Niu, Department of Respiratory Medicine, Xiangya Hospital, Central South University, 87 Xiangya Road, Changsha, Hunan 410008, P.R. China

E-mail: ruichaoniu@163.com

Key words: oxidized CaMKII, reactive oxygen species, asthma, chronic rhinosinusitis with nasal polyps, diabetic mellitus, cancer, acute ischemic stroke, cardiovascular substrate enzymes; ii) a central autoregulatory domain, which has different models of post-translational modifications and exhibits a suppressive effect by the mimic sequence of substrate enzyme; iii) a carboxy-terminal association domain, which has the subunits of oligomerization to produce holoenzyme and the variable sites of a variety of splice variants. Typically, the pseudosubstrate sequence of the autoregulatory domain maintains the basal kinase activity levels at 100-1,000-fold lower than the activity level stimulated by calcified calmodulin $\left(\mathrm{Ca}^{2+} / \mathrm{CaM}\right)(1)$.

The autoinhibitory region has residues that mimic a protein or nucleotide substrate. They interact with the catalytic domain and block the ATP- and substrate-binding pockets $(1,2)$. The binding of an allosteric activator, such as $\mathrm{Ca}^{2+} / \mathrm{CaM}$, alters the configuration of the autoinhibitory region, allowing access to the catalytic site (1). The sustained activation of the enzyme results in autophosphorylation at Thr287 (3). This leads to a 1,000-fold increase in the affinity of $\mathrm{CaM}$, and generates $\mathrm{Ca}^{2+} / \mathrm{CaM}$-independent enzyme activity (3). The activation of CaMKII further triggers the exchange of subunits between the holoenzymes, including the inactive ones, thus enabling the calcium-independent activation of new holoenzymes (4).

The activation of CaMKII is improved by reactive oxygen species (ROS), leading to cardiovascular disease, inflammation and cancer. The present review summarizes the current findings associated with the function of oxidized CaMKII (ox-CaMKII) in inflammatory diseases, including asthma, identifies important gaps in current knowledge and suggests novel approaches for future research (Table I).

\section{CaMKII is activated by ROS}

Erickson et al (5) was the first to observe that the activity and oxidation of CaMKII are enhanced in the myocardium under conditions of pressure, such as hypertrophy and infarction. Furthermore, the inhibition of CaMKII has been implicated in a reduction in the death rate of cardiomyocytes and an improvement of poor left ventricular remodeling. Of note, the level of ROS is decreased, suggesting that the inhibition of CaMKII hinders its oxidative role (5). ROS are produced by NADPH oxidase and mitochondria-oxidized methionine at 281 and 282, promoting autonomic enzyme activity (6). 
However, the suppressive role of pseudosubstrates requires the initial binding of $\mathrm{Ca}^{2+} / \mathrm{CaM}$ (7). The removal of ROS by inhibiting any of the pathways results in a marked decrease in the expression of oxidative CaMKII (ox-CaMKII) $(8,9)$. Several other factors, including hyperglycemia (8), excess intracellular $\mathrm{Na}^{+}$load (10), cardiac glycoside toxicity (11), and a variety of cytokines, including endotoxin (12), angiotensin II (Ang II) (13) and aldosterone (14), are able to enhance the expression of ox-CaMKII in the myocardium.

Methionine sulfoxide is the initial oxidation status of methionine, and methionine sulfoxide reductase A (MsrA) dynamically decreases the expression of ox-CaMKII by reducing the methionine residues, thus portraying the reversibility of the oxidative activation. Another novel mechanism underlying the autonomous activation of CaMKII can be observed in diabetes. Hyperglycemia causes the O-linked $\mathrm{N}$-acetylglucosamine modification of CaMKII at Ser279/280, activating it even at a low $\mathrm{Ca}^{2+}$ levels (15). $\beta$-adrenergic receptor signaling in cardiomyocytes results in the activation of CaMKII via a direct pathway, which involves the role of nitrosylation at C290. This NO-induced independent activation of CaMKII downstream to $\beta$-adrenergic signaling is responsible for increased sarcoplasmic reticulum-mediated $\mathrm{Ca}^{2+}$ leak and arrhythmogenesis. In fact, $\mathrm{NO}$ can also suppress the activation of CaMKII through the nitrosylation of C273, thus revealing the dual effects of NO on the activity of CaMKII (16-19). The phosphorylation of Ser26, a residue located within the ATP binding site of CaMKII $\gamma$, has been shown to shut off the activity of CaMKII in vascular tissues, thus terminating its sustained activation (20). Of note, methionine pairs are the most recent activators in vertebrate evolution, whereas S280, T287, C273, Ser26 and C290 are earlier activators, which also exist in invertebrate organisms. This indicates that M281/282 has physiological advantages in connection to the redox signal, but may have potential disadvantages in connection to excessive ROS caused by ox-CaMKII in several diseases.

Four isoforms $(\alpha, \beta, \gamma$ and $\delta$ ) of CaMKII have been identified in Homo sapiens (21). The alternative splicing of these isoforms results in the further expansion of CaMKII types by generating splice variants (22). These enzymes are differentially expressed in tissues. While CaMKII $\alpha$ and $\beta$ are predominantly present in the brain, the $\gamma$ and $\delta$ isoforms are expressed in various tissues $(21,23,24)$. The $\gamma$ isoform has been investigated most in the vasculature (25-27) and the $\delta$ isoform in cardiac tissues $(12,14,28-30)$. All isoforms share 89-93\% sequence similarity in their catalytic and autoregulatory domains (21). All isoforms have autophosphorylation sites, although their location differs marginally (Thr286 in $\alpha$ and Thr287 in $\beta, \gamma$ and $\delta$ ) in the core regulatory and CaM-binding domains (Thr305/306 in $\alpha$ and Thr306/307 in $\beta, \gamma$ and $\delta$ ). Whereas all other isoforms have paired methionine residues within the self-inhibiting areas of the regulatory regions, the $\alpha$ isoform has cysteine-methionine residues, all of which are able to autonomously activate $\mathrm{Ca}^{2+} / \mathrm{CaM}$ during oxidation (5). In its resting state, without $\mathrm{Ca}^{2+} / \mathrm{CaM}$ binding, CaMKII is almost inactive, as the self-inhibitory region of the regulatory and catalytic domains combine and inhibit the release of ATP. However, M281/282 oxidation transforms the hydrophobic residues into hydrophilic species, which tend to bind to become involved in the substrate binding site, even without $\mathrm{Ca}^{2+} / \mathrm{CaM}$ binding (6). The following summarizes the role of ox-CaMKII in inflammatory diseases.

\section{Role of ox-CaMKII in inflammatory diseases}

Ox-CaMKII promotes acute ischemic stroke. Acute ischemic stroke is the process of rapid loss of neurological function as a result of insufficient blood flow to affected brain areas. According to the model of transient cerebral ischemia, ROS aggravate the severity of stroke, resulting in neurological disorders (31). It has been reported that MsrA, an antioxidant enzyme that can reverse methionine oxidation, decreased the ROS-induced activation of nuclear factor (NF)- $\mathrm{kB}$ in endothelial cells by inhibiting the oxidation of methionine residues in the regulatory region of CaMKII (32). CaMKII is the upstream protein kinase of NF- $\kappa B$, which has been shown to modulate the activation of NF- $\kappa B$ in myocardial ischemia/reperfusion injury $(5,12)$. The treatment of human umbilical vein endothelial cells (HUVECs) with $\mathrm{H}_{2} \mathrm{O}_{2}$ leads to CaMKII Met281/282 oxidation. This means that ROS can lead to the oxidation and activation of CaMKII. To confirm that the stimulatory effect of CaMKII Met281/282 oxidation can induce the activation of NF- $\mathrm{BB}$ in endothelial cells, HUVECs were infected with oxidation-resistant CaMKII tandem mutants with methionine at Met281/282. The results showed that the overexpression of CaMKII at Met281/282 almost inhibited the $\mathrm{H}_{2} \mathrm{O}_{2}$-induced activation of NF- $\mathrm{KB}$ to inhibit cerebral ischemia/reperfusion injury. The administration of the CaMKII inhibitor KN-93 also protected $\mathrm{MsrA}^{-/-}$mice from exacerbated neurological deficits following cerebral ischemia/reperfusion injury. In conclusion, with the assistance of ROS, ox-CaMKII can cause $\mathrm{NF}-\kappa \mathrm{B}$ to aggravate neurovascular inflammation and brain tissue injury in ischemic stroke (32).

Ox-CaMKII promotes cardiovascular disease. The sources of ROS within cardiac myocytes include mitochondria, NADPH oxidase, xanthine oxidase and uncoupled nitric oxide synthases (33). The production of ROS increases in myocardial infarction (MI) and heart failure (34). Enhanced oxidative stress leads to NF- $\mathrm{BB}$-mediated cytokine release and contributes to inflammation (35). It also causes myocardial contractile dysfunction and remodeling, leading to heart failure (36). Ox-CaMKII levels are also elevated in conditions of increased oxidative stress, including MI $(5,12,14)$, arrhythmia $(11,14,37,38)$, vascular smooth muscle migration and cell apoptosis $(14,39,40)$, and acute myocardial ischemia (41). The inhibition of ox-CaMKII blunts the expression of inflammatory genes (6).

The expression of ox-CaMKII was found to be enhanced following Ang II exposure in a model of MI, in addition to myocardial death, dysfunction and unfavorable left ventricular function (5). The level of ox-CaMKII is particularly enhanced during the initial $24 \mathrm{~h}$ following MI, depending on the generation of ROS by regulating innate immune adaptors, including Toll-like receptor 4 and myeloid differentiation factor- 88 (MyD88) (12). However, MyD88-deficient mice exhibited reduced expression of ox-CaMKII, myocardial hypertrophy and increased mortality due to MI, and reduced transcription levels (12). Furthermore, infusion aldosterone enhances myocardial ROS and ox-CaMKII and leads to MI, and 
Table I. Ox-CamKII promotes the development of multiple diseases.

\begin{tabular}{lll}
\hline Author, year & Disease & Relevant functions \\
\hline
\end{tabular}

Gu et al, 2016

Erickson et al, 2008; Singh et al, 2012; $\quad$ MI

He et al, 2011

Ho et al, 2014; Swaminathan et al, 2011; Arrhythmias

Purohit et al, 2013; Wagner et al, 2011

He et al, 2011; Zhu et al, 2014;

Scott et al, 2012

Vascular disease

Rajtik et al, 2016

Acute myocardial ischemia/reperfusion injury

Luo et al, 2013

Diabetic mellitus

Hart et al, 2015

Breast cancer

Qu et al, 2017

Asthma

Wang et al, 2018

CRSwNP
$\mathrm{Ox}-\mathrm{CaMKII}$ can induce nuclear factor- $\kappa \mathrm{B}$ to

exacerbate neurovascular inflammation and cerebral tissue damage in ischemic stroke.

Ox-CaMKII is increased by Ang II and

aldosterone and induces MI

$\mathrm{Ox}-\mathrm{CaMKII}$ is increased in atrial fibrillation in the presence of Ang II and a key mediator of the cardiac glycoside reactive oxygen species-induced arrhythmogenic effects on myocyte $\mathrm{Ca}^{2+}$ handling.

Ox-CaMKII is involved in vascular smooth muscle responses to injury and may provide feedback information for cellular redox balance.

Ox-CaMKII induces the development of acute myocardial ischemia/reperfusion injury.

Ox-CaMKII causes higher mortality rates in diabetic patients with MI.

Ox-CaMKII as a key signal for breast cancer cells and CaMKII inhibition may have therapeutic benefit in types of cancer relying on glycolysis for selective advantage over non-tumor cells

Ox-CaMKII is predominantly present in the epithelium (9) Ox-CaMKII significantly promotes asthma through mast cell activation.

ox-CaMKII is involved in KYN/AhR signaling-mediated mast cell activation in CRSwNP. 
activation of CaMKII decreased VSMC migration and apoptosis. Furthermore, the mRNA levels of CaMKII $\gamma$ and $-\delta$ were clearly enhanced in MM-VV $\delta$ mice, when compared with WT VSMCs, suggesting that the expression of CaMKII is regulated by ROS $(14,39)$. These findings show that ox-CaMKII is involved in the response of damaged vascular smooth muscle and suggests that ox-CaMKII is able to supply the feedback signaling pathway to balance the intercellular redox response.

Ox-CaMKII is also critical in the development of acute myocardial ischemia/reperfusion injury. Ox-CaMKIID is downregulated at the end of reperfusion compared with levels in ischemia. The NADPH oxidase 2-ox-CAMKIII signaling pathway is unlikely to be involved in the cardioprotective obstruction of Ang II receptor type 1 activation, which can be inhibited with CaMKII (41).

Ox-CaMKII promotes diabetes mellitus. Diabetes mellitus is a major public health concern that influences $>8 \%$ of the US population $(42,43)$. MI is one of the most important causes of mortality in patients with diabetes, with a study reporting that MI-associated mortality rates in patients with diabetes are twice those of patients without (42). It has been reported that diabetic patients with MI exhibit higher ox-CaMKII levels in their right atrium, compared with non-diabetic patients with MI. In models of diabetic mice with MI, increased SAN cell death and fibrosis were present, which was prevented by CaMKII inhibition and the oxidation-resistant CaMKII mutant (MM-VV), highlighting the importance of ox-CaMKII as a cause of higher mortality rates in diabetic patients with MI (8). The elevated expression of ox-CaMKII in diabetes is due to increased mitochondrial superoxide production and hyperglycemia-induced ROS production (8). In MM-VV and WT mice treated with streptozotocin and injected with ROS-targeted inhibitor, the expression of ox-CaMKII was decreased, the survival rate of pacemaker cells was enhanced, no significant difference was observed in the heart rate, and diabetes-attributable mortality was reduced following MI (8), suggesting that the ROS-ox-CaMKII signaling pathway can induce an increase in sudden mortality in diabetic patients following MI.

Ox-CaMKII promotes cancer. The overactivation of CaMKII has been shown to promote several types of cancer. CAMKII serves an important role in cancer cell proliferation, differentiation and survival, and has been implicated in various types of cancer, including lung (44), breast $(45,46)$, prostate (47) and colon cancer $(48,49)$. Cancer cells favor glycolysis in low-oxygen environments to ensure survival, a phenomenon commonly known as the Warburg effect (50). Evidence shows that CaMKII is a key ROS sensor and that ox-CaMKII facilitates the activation of AMPK phosphorylation at Thr172 (51). The use of a CaMKII inhibitor resulted in the dephosphorylation of AMPK, decreased glycolysis and reduced steady-state ATP levels. It also markedly reduced the survivability of cells with a high expression of MnSOD (51). A comparison between KN-93, a CaMKII inhibitor and chemotherapeutic agent, paclitaxel and fluorouracil in aggressive breast cancer revealed a greater efficacy of KN-93 (51).

Ox-CaMKII promotes asthma. Asthma is a chronic airway inflammatory disease,typically featuring airway hyperreactivity and reversible airflow obstruction, influx and activation of inflammatory cells, and release of inflammatory cytokines ultimately leading to airway remodeling $(52,53)$. It is a major public health burden and is now considered one of the most common chronic disorders worldwide (54). The total number of individuals with asthma may be as high as $334,000,000$. Asthma affects $\sim 8.5 \%$ of the total US population and, in 2007, the estimated cost related to medical expenses and lost work due to asthma was $\$ 56,000,000,000$ annually (55). High-oxygen environments, large airway surface area and rich blood predispose lungs to oxidative stress. The two primary sources of ROS in the lungs are environmental, including gaseous and particulate air pollution, and cellular. The main sources of cellular ROS are mitochondrial respiration, NADPH oxidase and the xanthine/xanthine oxidase system in epithelial and inflammatory cells (52). Increased levels of ROS have been detected in the gases exhaled by patients with asthma, compared with healthy controls (56). ROS induce goblet cell proliferation and metaplasia, and mucus hypersecretion by increasing the gene and protein expression of MUC5AC (57). They are also implicated in eosinophil recruitment and airway hyperresponsiveness (58). However, the exact molecular mechanisms linking the ability of ROS to cause several phenotypic features of asthma remain to be fully elucidated. The role of ox-CaMKII has been widely examined in heart diseases, and the presence of both increased ROS and ox-CaMKII levels in the diseased myocardia has prompted investigations med at identifying a potential link between the two in airways.

It is well-known that the level of ROS is elevated in asthma. CaMKII is a critical ROS sensor. A study showed that smooth muscle CaMKIIס facilitates allergen-induced airway hyperresponsiveness and inflammation (59). However, detection of ox-CaMKII in the bronchial epithelium and smooth muscle revealed that it mainly exists in the epithelium rather than in the smooth muscle, and that ox-CaMKII is enhanced in patients with severe asthma compared to total CaMKII (9). Increased ox-CaMKII levels were also detected in ovalbumin (OVA)-treated mice compared with vehicle-treated mice; there was no marked difference in total CaMKII. In addition, the expression of ox-CaMKII was found to be elevated further in patients with severe asthma compared with either patients with mild asthma or healthy individuals, suggesting that ox-CaMKII can be used to not only diagnose asthma, but also determine its severity (9). The P47 gene encodes NADPH oxidase, which is required for the production of ROS (60). $\mathrm{P} 7^{--}$OVA mice exhibited a lower expression of ROS and ox-CaMKII compared with WT OVA mice. These findings indicated the crucial role of P47-induced NADPH oxidase in ROS generation and the production of ox-CaMKII. Compared with WT OVA mice, $\mathrm{P} 47^{--}$mice exhibited a significant decline in goblet cell proliferation, bronchial epithelium thickness, more positive MUC5AC airway cells, mRNA expression of MUC5AC, airway eosinophilia and one of the eosinophil chemoattractant molecules (Ccl-11), when compared with WT OVA mice. These changes were similar to those observed following corticosteroid use (61). These results indicated that NADPH oxidase-derived ROS serve a vital role in the phenotypic features of asthma and CaMKII oxidation. The antioxidant enzyme MsrA reduces CaMKII by reversing the paired oxidation of Met281/282 $(32,62)$. Compared 


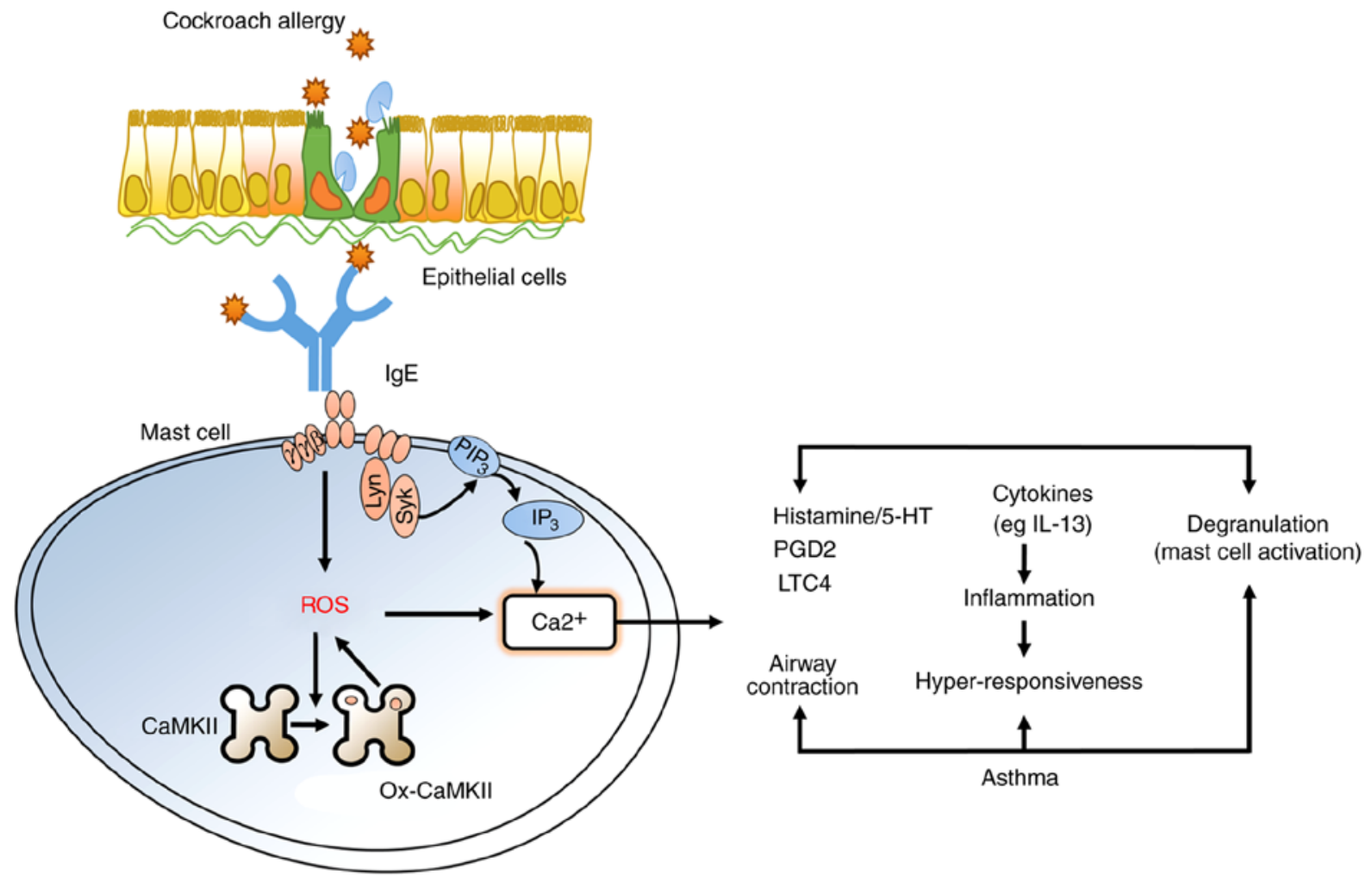

Figure 1. Ox-CaMKII promotes asthma through the activation of mast cells. CRE induces the generation of ROS to modulate the ox-CaMKII activation of mast cells, which is dependent on intracellular $\mathrm{Ca}^{2+}$ generation. In particular, ox-CaMKII regulates mast cell degranulation, histamine release, and the expression of LTC4 and IL-13. Ox-CaMKII, oxidized CaMKII; CRE, cockroach allergen; ROS, reactive oxygen species; PGD4, prostaglandin 4; LTC4, leukotriene C4; IL-13, interleukin 13

with WT OVA mice, $\mathrm{MsrA}^{-/-}$OVA mice exhibited a marked enhancement in goblet cell proliferation, bronchial epithelium thickness, positive MUC5AC airway cells, mRNA expression of MUC5AC, airway eosinophilia and Ccl-11, when compared with WT OVA mice (9). NF- $\mathrm{BB}$ is a major modulator of asthma progression, inflammatory signaling and expression of MUC5AC (63). OVA mice expressing NF- $\kappa B$ reporter exhibited a notable increase in NF- $\mathrm{KB}$ levels compared with saline-treated mice. However, the genetic manipulation of pathways for ROS generation and CaMKII oxidation affected the levels of NF- $\kappa B$. MsrA ${ }^{-/}$exhibited an increase in NF- $\kappa \mathrm{B}$, indicating that the effects of NF- $\kappa$ B signaling pathways lie downstream of ROS and ox-CaMKII in the pathogenesis of asthma (9).

The $\gamma$-aminobutyric acid type A (GABA) receptors present in the pulmonary epithelial cells exhibit a markedly increased expression when sensitized and challenged with OVA. Chloride currents $\left(\mathrm{I}_{\mathrm{Cl}}\right)$ activity in the bronchial epithelium is mostly due to these GABA receptors; an increase in $\mathrm{I}_{\mathrm{Cl}}$ activity was observed in OVA mice compared with that in the tracheal epithelial cells of saline-treated mice. The addition of GABA receptor antagonist picrotoxin and CaMKII inhibitor KN-93 (8) significantly reduced $\mathrm{I}_{\mathrm{Cl}}$ activity in OVA mice but did not further reduce activity if both were introduced in the mice simultaneously, suggesting the existence of a pathway for $\mathrm{I}_{\mathrm{Cl}}$-mediated goblet cell hyperplasia. $\mathrm{I}_{\mathrm{Cl}}$ activity was also enhanced considerably more in cells overexpressing ox-CaMKII than in cells expressing MM-VV CaMKII mutant or those expressing CaMKII when $\mathrm{H}_{2} \mathrm{O}_{2}$ was added.
OVA-induced $\mathrm{I}_{\mathrm{Cl}}$ activity was decreased in $\mathrm{P} 47^{--}$OVA mice. This indicates the importance of ox-CaMKII derived from NADPH oxidase-derived ROS in $\mathrm{I}_{\mathrm{Cl}}$ activity. WT OVA mice exhibited a marked enhancement in airway hyper-reactivity compared with saline-treated controls. Transgenic CaMKII with inhibitory peptide in OVA epi-AC3-I mice reduced goblet cell proliferation and bronchial epithelium thickness, and increased the mRNA expression of MUC5AC, airway eosinophilia, airway hyperreactivity and $\mathrm{I}_{\mathrm{Cl}}$ activity, compared with the same features in WT OVA mice (9).

Ox-CaMKII promotes asthma through the activation of mast cells. It has been shown that ox-CaMKII can be used to not only diagnose asthma, but also determine its severity. In the present review, the function of ox-CaMKII in asthma and its underlying mechanism were further examined. For the first time, the oxidant-resistant CaMKII MM-VV was used to construct asthma using M281/282 valines (MM-VVס) in the isotype genetic background of isoform $\delta$ mutation in knock-in mice (64). Cockroach allergen (CRE)-treated MM-VV $\delta$ mice exhibited considerably decreased airway inflammation, lower airway resistance and lower concentrations of Th2 cytokines, compared with CRE-treated WT mice. The role of ROS and/or ox-CaMKII in the activation of mast cells remains to be elucidated. It was demonstrated that ox-CaMKII modulates the migration and activation of mast cells in the lung tissues of CRE-exposed mice, thus promoting the occurrence of asthma.

In vitro, the enhanced expression of mitochondrial ROS was identified in OVA-treated bone marrow mast cells (BMMCs) 
compared with that in controls. Similar to mitochondrial ROS, the expression of intracellular ROS was markedly decreased in MM-VVס BMMCs, compared with that in the WT group, which is consistent with the previous observations of increased ROS production in OVA-activated mast cells (65). It is noteworthy that MM-VV $\delta$ mast cells exhibited a decline in mast cell degranulation following OVA stimulation, decreased histamine release, and decreased expression of leukotriene $\mathrm{C} 4$ and interleukin (IL)-13, indicating that the promotion of mast cell activation by ox-CaMKII relies on intracellular $\mathrm{Ca}^{2+}$ generation.

KN-93 inhibited OVA-induced asthma in a previous study (9), therefore, KN-93 was used to further investigate the mechanism underlying the effect of CaMKII in the activation of mast cells. The results showed that the dose-dependent suppression of IgE regulates mast cell granules and the secretion of IL-13 in BMMCs and human mast cells (HMC-1), suggesting that reducing the expression of CaMKII protects against allergies caused by mast cell activation. The present review is the first time, to the best of our knowledge, that the CRE-ROS-ox-CaMKII axis has been linked to the modulation of mast cell activation following the progress of asthma and allergies (Fig. 1).

Role of ox-CaMKII in chronic rhinosinusitis with nasal polyps $(C R S w N P)$. CRSwNP is an inflammatory sinonasal disease with Th2-skewed eosinophilic inflammation in mast cells, which is vital in regulating the environmental antigen-induced pathogenesis of CRSwNP (66). It is known that aryl hydrocarbon receptor (AhR), a ligand-induced transcriptional factor, triggers the production of ROS and the $\mathrm{Ca}^{2+}$-reliant activation of mast cells (65). AhR can also detect endogenous tryptophan metabolites produced by kynurenine $(\mathrm{KYN})$ or indoleamine 2,3-dioxygenase and tryptophan 2,3-dioxygenas, which modulate the activation of mast cells $(67,68)$. Our previous study examined whether ROS, serving as the upstream of ox-CaMKII, aggravated CRE-mediated lung inflammation through activating mast cells (64). However, the role of ox-CaMKII associated with the KYN/AhR signaling pathway induced by mast cell activation in CRSwNP remains unknown. Another study reported that KYN activated AhR signaling in CRSwNP, and that AhR regulated the generation of ROS and activation of mast cells. Furthermore, the expression of ox-CaMKII was considerably increased in the airway epithelial cells and cells infiltrating the sinonasal mucosa. In addition, ox-CaMKII was shown to be involved in the KYN/AhR signaling pathway and modulate the activation of mast cells in CRSwNP. These studies suggested an important function of the KYN/AhR axis in mediating the activation of mast cells through ox-CaMKII in the pathogenesis of CRSwNP, although further elucidation is required (69).

\section{Conclusions}

Ox-CaMKII is a vital sensor of ROS in different diseases, which regulates calcium signals and the ROS signaling pathway in several subcellular loci. In recent years, ox-CaMKII has been shown to affect immune regulation, particularly through regulating the activation of mast cells in asthma and CRSwNP. However, global antioxidant treatments are mostly unsuccessful. It is imperative to obtain an understanding of the mechanisms through which the ROS-modulated signaling pathway leads to the development of related diseases. Our recent studies and those of others have supported the hypothesis that CaMKII can mediate an effective antioxidant treatment for asthma and CRSwNP $(64,69)$. Studies involving MM-VV mice may assist in assessing the therapeutic potential of ox-CaMKII.

\section{Acknowledgements}

Not applicable.

\section{Funding}

This work was supported in part by the National Youth Science Foundation of China (grant no. 81802278 to QJJ).

\section{Availability of data and materials}

Data sharing is not applicable to this article, as no datasets were generated or analyzed during the current study.

\section{Authors' contributions}

JJQ and RCN wrote the manuscript; JJQ and QHM collected the references and modified the manuscript; QHM analyzed the data; RCN designed the manuscript and approved the final manuscript.

\section{Ethics approval and consent to participate}

Not applicable.

\section{Patient consent for publication}

Not applicable.

\section{Competing interests}

The authors declare that they have no competing interests.

\section{References}

1. Hudmon A and Schulman H: Structure-function of the multifunctional $\mathrm{Ca}^{2+} /$ calmodulin-dependent protein kinase II. Biochem J 364: 593-611, 2002.

2. Rosenberg OS, Deindl S, Sung RJ, Nairn AC and Kuriyan J: Structure of the autoinhibited kinase domain of CaMKII and SAXS analysis of the holoenzyme. Cell 123: 849-860, 2005.

3. Erickson JR, He BJ, Grumbach IM and Anderson ME: CaMKII in the cardiovascular system: Sensing redox states. Physiol Rev 91: 889-915, 2011.

4. Stratton M, Lee IH, Bhattacharyya M, Christensen SM, Chao LH, Schulman H, Groves JT and Kuriyan J: Activation-triggered subunit exchange between CaMKII holoenzymes facilitates the spread of kinase activity. Elife 3: e01610, 2013.

5. Erickson JR, Joiner ML, Guan X, Kutschke W, Yang J, Oddis CV, Bartlett RK, Lowe JS, O'Donnell SE, Aykin-Burns N, et al: A dynamic pathway for calcium-independent activation of CaMKII by methionine oxidation. Cell 133: 462-474, 2008.

6. Brookes PS, Yoon Y, Robotham JL, Anders MW and Sheu SS: Calcium, ATP, and ROS: A mitochondrial love-hate triangle. Am J Physiol Cell Physiol 287: C817-C833, 2004. 
7. Nickel AG, Kohlhaas M, Bertero E, Wilhelm D, Wagner M Sequeira V, Kreusser MM, Dewenter M, Kappl R, Hoth M, et al: CaMKII does not control mitochondrial $\mathrm{Ca}^{2+}$ uptake in cardiac myocytes. J Physiol: Feb 16, 2019 (Epub ahead of print). doi: 10.1113/JP276766.

8. Luo M, Guan X, Luczak ED, Lang D, Kutschke W, Gao Z, Yang J, Glynn P, Sossalla S, Swaminathan PD, et al: Diabetes increases mortality after myocardial infarction by oxidizing CaMKII. J Clin Invest 123: 1262-1274, 2013

9. Sanders PN, Koval OM, Jaffer OA, Prasad AM, Businga TR, Scott JA, Hayden PJ, Luczak ED, Dickey DD, Allamargot C, et al: CaMKII is essential for the proasthmatic effects of oxidation. Sci Transl Med 5: 195ra97, 2013.

10. Viatchenko-Karpinski S Kornyeyev D,El-Bizri N, Budas G, Fan P, Jiang Z, Yang J, Anderson ME, Shryock JC, Chang CP, et al: Intracellular $\mathrm{Na}^{+}$overload causes oxidation of CaMKII and leads to $\mathrm{Ca}^{2+}$ mishandling in isolated ventricular myocytes. J Mol Cell Cardiol 76: 247-256, 2014.

11. Ho HT, Liu B, Snyder JS, Lou Q, Brundage EA, Velez-Cortes F, Wang H, Ziolo MT, Anderson ME, Sen CK, et al: Ryanodine receptor phosphorylation by oxidized CaMKII contributes to the cardiotoxic effects of cardiac glycosides. Cardiovasc Res 101: 165-174, 2014.

12. Singh MV, Swaminathan PD, Luczak ED, Kutschke W, Weiss RM and Anderson ME: MyD88 mediated inflammatory signaling leads to CaMKII oxidation, cardiac hypertrophy and death after myocardial infarction. J Mol Cell Cardiol 52: $1135-1144,2012$

13. Swaminathan PD, Purohit A, Soni S, Voigt N, Singh MV, Glukhov AV, Gao Z, He BJ, Luczak ED, Joiner ML, et al: Oxidized CaMKII causes cardiac sinus node dysfunction in mice. J Clin Invest 121: 3277-3288, 2011.

14. He BJ, Joiner ML, Singh MV, Luczak ED, Swaminathan PD, Koval OM, Kutschke W, Allamargot C, Yang J, Guan X, et al: Oxidation of CaMKII determines the cardiotoxic effects of aldosterone. Nat Med 17: 1610-1618, 2011.

15. Erickson JR, Pereira L, Wang L, Han G, Ferguson A, Dao K, Copeland RJ, Despa F, Hart GW, Ripplinger CM and Bers DM: Diabetic hyperglycaemia activates CaMKII and arrhythmias by O-linked glycosylation. Nature 502: 372-376, 2013.

16. Gutierrez DA, Fernandez-Tenorio M, Ogrodnik J and Niggli E: NO-dependent CaMKII activation during $\beta$-adrenergic stimulation of cardiac muscle. Cardiovasc Res 100: 392-401, 12013

17. Coultrap SJ and Bayer KU: Nitric oxide induces $\mathrm{Ca}^{2+}$-independent activity of the $\mathrm{Ca}^{2+} / \mathrm{calmodulin}$-dependent protein kinase II (CaMKII). J Biol Chem 289: 19458-19465, 2014.

18. Curran J, Tang L, Roof SR, Velmurugan S, Millard A, Shonts S, Wang H, Santiago D, Ahmad U, Perryman M, et al: Nitric oxide-dependent activation of CaMKII increases diastolic sarcoplasmic reticulum calcium release in cardiac myocytes in response to adrenergic stimulation. PLoS One 9 e87495, 2014

19. Erickson JR, Nichols CB, Uchinoumi H, Stein ML, Bossuyt J and Bers DM: S-Nitrosylation induces both autonomous activation and inhibition of Calcium/Calmodulin-dependent protein kinase II $\delta$. J Biol Chem 290: 25646-25656, 2015.

20. Yilmaz M, Gangopadhyay SS, Leavis P, Grabarek Z and Morgan KG: Phosphorylation at $\mathrm{Ser}^{26}$ in the ATP-binding site of $\mathrm{Ca}^{2+} /$ calmodulin-dependent kinase II as a mechanism for switching off the kinase activity. Biosci Rep 33: e00024, 2013.

21. Tobimatsu $\mathrm{T}$ and Fujisawa H: Tissue-specific expression of four types of rat calmodulin-dependent protein kinase II mRNAs. J Biol Chem 264: 17907-17912, 1989.

22. Tombes RM and Krystal GW: Identification of novel human tumor cell-specific CaMK-II variants. Biochim Biophys Acta 1355: 281-292, 1997

23. Takaishi T, Saito $\mathrm{N}$ and Tanaka C: Evidence for distinct neuronal localization of gamma and delta subunits of $\mathrm{Ca}^{2+} / \mathrm{calmod}-$ ulin-dependent protein kinase II in the rat brain. J Neurochem 58 1971-1974, 1992.

24. Bayer KU, Löhler J, Schulman H and Harbers K: Developmental expression of the CaM kinase II isoforms: Ubiquitous gammaand delta-CaM kinase II are the early isoforms and most abundant in the developing nervous system. Brain Res Mol Brain Res 70: 147-154, 1999.

25. Kim I, Je HD, Gallant C, Zhan Q, Riper DV, Badwey JA, Singer HA and Morgan KG: $\mathrm{Ca}^{2+}$-calmodulin-dependent protein kinase II-dependent activation of contractility in ferret aorta. J Physiol 526: 367-374, 2000.
26. Gangopadhyay SS, Barber AL, Gallant C, Grabarek Z, Smith JL and Morgan KG: Differential functional properties of calmodulin-dependent protein kinase IIgamma variants isolated from smooth muscle. Biochem J 372: 347-357, 2003

27. Marganski WA, Gangopadhyay SS, Je HD, Gallant C and Morgan KG: Targeting of a novel $\mathrm{Ca}^{+2} /$ calmodulin-dependent protein kinase II is essential for extracellular signal-regulated kinase-mediated signaling in differentiated smooth muscle cells. Circ Res 97: 541-549, 2005.

28. Guo T, Zhang T, Ginsburg KS, Mishra S, Brown JH and Bers DM: CaMKIIIC slows Ca]i decline in cardiac myocytes by promoting Ca sparks. Biophys J 102: 2461-2470, 2012.

29. Mishra S, Ling H, Grimm M, Zhang T, Bers DM and Brown JH: Cardiac hypertrophy and heart failure development through Gq and CaM kinase II signaling. J Cardiovasc Pharmacol 56: 598-603, 2010

30. Singh MV, Kapoun A, Higgins L, Kutschke W, Thurman JM, Zhang R, Singh M, Yang J, Guan X, Lowe JS, et al: $\mathrm{Ca}^{2+} / \mathrm{calmod}^{-}$ ulin-dependent kinase II triggers cell membrane injury by inducing complement factor $\mathrm{B}$ gene expression in the mouse heart. J Clin Invest 119: 986-996, 2009.

31. Crack PJ, Taylor JM, Ali U, Mansell A and Hertzog PJ: Potential contribution of NF-kappaB in neuronal cell death in the glutathione peroxidase-1 knockout mouse in response to ischemia-reperfusion injury. Stroke 37: 1533-1538, 2006.

32. Gu SX, Blokhin IO, Wilson KM, Dhanesha N, Doddapattar P, Grumbach IM, Chauhan AK and Lentz SR: Protein methionine oxidation augments reperfusion injury in acute ischemic stroke. JCI Insight 1: pii: e86460, 2016.

33. Kimura W, Muralidhar S, Canseco DC, Puente B, Zhang CC, Xiao F, Abderrahman YH and Sadek HA: Redox signaling in cardiac renewal. Antioxid Redox Signal 21: 1660-1673, 2014.

34. Fraccarollo D, Galuppo P, Neuser J, Bauersachs J and Widder JD: Pentaerythritol tetranitrate targeting myocardial reactive oxygen species production improves left ventricular remodeling and function in rats with ischemic heart failure. Hypertension 66 : 978-987, 2015.

35. Frantz S, Brandes RP, Hu K, Rammelt K, Wolf J, Scheuermann H, Ertl $G$ and Bauersachs J: Left ventricular remodeling after myocardial infarction in mice with targeted deletion of the NADPH oxidase subunit gp91PHOX. Basic Res Cardiol 101: 127-132, 2006

36. Murdoch CE, Zhang M, Cave AC and Shah AM: NADPH oxidase-dependent redox signalling in cardiac hypertrophy, remodelling and failure. Cardiovasc Res 71: 208-215, 2006.

37. Purohit A, Rokita AG, Guan X, Chen B, Koval OM, Voigt N, Neef S, Sowa T, Gao Z, Luczak ED, et al: Oxidized $\mathrm{Ca}(2+) /$ calmodulin-dependent protein kinase II triggers atrial fibrillation. Circulation 128: 1748-1757, 2013.

38. Wagner S, Ruff HM, Weber SL, Bellmann S, Sowa T, Schulte T, Anderson ME, Grandi E, Bers DM, Backs J, et al: Reactive oxygen species-activated $\mathrm{Ca} / \mathrm{calmodulin}$ kinase II $\delta$ is required for late $\mathrm{I}(\mathrm{Na})$ augmentation leading to cellular $\mathrm{Na}$ and $\mathrm{Ca}$ overload. Circ Res 108: 555-565, 2011.

39. Zhu LJ, Klutho PJ, Scott JA, Xie L, Luczak ED, Dibbern ME, Prasad AM, Jaffer OA, Venema AN, Nguyen EK, et al: Oxidative activation of the $\mathrm{Ca}(2+) /$ calmodulin-dependent protein kinase II (CaMKII) regulates vascular smooth muscle migration and apoptosis. Vascul Pharmacol 60: 75-83, 2014.

40. Scott JA, Xie L, Li H, Li W, He JB, Sanders PN, Carter AB, Backs J, Anderson ME and Grumbach IM: The multifunctional $\mathrm{Ca}^{2+} /$ calmodulin-dependent kinase II regulates vascular smooth muscle migration through matrix metalloproteinase 9. Am J Physiol Heart Circ Physiol 302: H1953-H1964, 2012.

41. Rajtik T, Carnicka S, Szobi A, Giricz Z, O-Uchi J, Hassova V, Svec P, Ferdinandy P, Ravingerova T and Adameova A: Oxidative activation of CaMKIIס in acute myocardial ischemia/reperfusion injury: A role of angiotensin AT1 receptor-NOX2 signaling axis. Eur J Pharmacol 771: 114-122, 2016.

42. Knowler WC, Barrett-Connor E, Fowler SE, Hamman RF, Lachin JM, Walker EA and Nathan DM: Reduction in the incidence of type 2 diabetes with lifestyle intervention or met-formin. N Engl J Med 346: 393-403, 2002.

43. Donahoe SM, Stewart GC, McCabe CH, Mohanavelu S, Murphy SA, Cannon CP and Antman EM: Diabetes and mortality following acute coronary syndromes. JAMA 298: 765-775, 2007. 
44. Chai S, Qian Y, Tang J, Liang Z, Zhang M, Si J, Li X, Huang W, Xu R and Wang K: Retracted: $\mathrm{Ca}(2+) /$ calmodulin-dependent protein kinase II $\gamma$, a critical mediator of the NF- $\kappa \mathrm{B}$ network, is a novel therapeutic target in non-small cell lung cancer. Cancer Lett 344: 119-128, 2014.

45. Britschgi A, Bill A, Brinkhaus H, Rothwell C, Clay I, Duss S, Rebhan M, Raman P, Guy CT, Wetzel K, et al: Calcium-activated chloride channel ANO1 promotes breast cancer progression by activating EGFR and CAMK signaling. Proc Natl Acad Sci USA 110: E1026-E1034, 2013.

46. Kim JH, Kim TW and Kim SJ: Downregulation of ARFGEF1 and CAMK2B by promoter hypermethylation in breast cancer cells. BMB Rep 44: 523-528, 2011.

47. Wang T, Guo S, Liu Z, Wu L, Li M, Yang J, Chen R, Liu X, Xu H, Cai S, et al: CAMK2N1 inhibits prostate cancer progression through androgen receptor-dependent signaling. Oncotarget 5: 10293-10306, 2014.

48. Wang C, Li N, Liu X, Zheng Y and Cao X: A novel endogenous human CaMKII inhibitory protein suppresses tumor growth by inducing cell cycle arrest via p27 stabilization. J Biol Chem 283 : 11565-11574, 2008.

49. Jing Z, Sui X, Yao J, Xie J, Jiang L, Zhou Y, Pan H and Han W: SKF-96365 activates cytoprotective autophagy to delay apoptosis in colorectal cancer cells through inhibition of the calcium/CaMKII $\gamma / \mathrm{AKT}$-mediated pathway. Cancer Lett 372: 226-238, 2016.

50. Bhat PJ, Darunte L, Kareenhalli V, Dandekar J and Kumar A: Can metabolic plasticity be a cause for cancer? Warburg-Waddington legacy revisited. Clin Epigenetics 2: 113-122, 2011.

51. Hart PC, Mao M, de Abreu AL, Ansenberger-Fricano K, Ekoue DN, Ganini D, Kajdacsy-Balla A, Diamond AM, Minshall RD, Consolaro ME, et al: MnSOD upregulation sustains the Warburg effect via mitochondrial ROS and AMPK-dependent signalling in cancer. Nat Commun 6: 6053, 2015.

52. Kirkham P and Rahman I: Oxidative stress in asthma and COPD: Antioxidants as a therapeutic strategy. Pharmacol Ther 111: 476-494, 2006

53. Jaffer OA, Carter AB, Sanders PN, Dibbern ME, Winters CJ, Murthy S, Ryan AJ, Rokita AG, Prasad AM, Zabner J, et al: Mitochondrial-targeted antioxidant therapy decreases transforming growth factor- $\beta$-mediated collagen production in a murine asthma model. Am J Respir Cell Mol Biol 52: 106-115, 2015.

54. Anandan C, Nurmatov U, van Schayck OC and Sheikh A: Is the prevalence of asthma declining? Systematic review of epidemiological studies. Allergy 65: 152-167, 2010.

55. Lambrecht BN and Hammad $\mathrm{H}$ : The immunology of asthma. Nat Immunol 16: 45-56, 2015

56. Huang SK, Zhang Q, Qiu Z and Chung KF: Mechanistic impact of outdoor air pollution on asthma and allergic diseases. J Thorac Dis 7: 23-33, 2015.
57. Casalino-Matsuda SM, Monzón ME and Forteza RM: Epidermal growth factor receptor activation by epidermal growth factor mediates oxidant-induced goblet cell metaplasia in human airway epithelium. Am J Respir Cell Mol Biol 34: 581-591, 2006.

58. Abdala-Valencia H, Earwood J, Bansal S, Jansen M, Babcock G, Garvy B, Wills-Karp M and Cook-Mills JM: Nonhematopoietic NADPH oxidase regulation of lung eosinophilia and airway hyperresponsiveness in experimentally induced asthma. Am J Physiol Lung Cell Mol Physiol 292: L1111-L1125, 2007.

59. Spinelli AM, Liu Y, Sun LY, González-Cobos JC, Backs J, Trebak M and Singer HA: Smooth muscle CaMKIId promotes allergen-induced airway hyper-responsiveness and inflammation. Pflugers Arch 467: 2541-2554, 2015

60. Li JM, Mullen AM, Yun S, Wientjes F, Brouns GY, Thrasher AJ and Shah AM: Essential role of the NADPH oxidase subunit $\mathrm{p} 47$ (phox) in endothelial cell superoxide production in response to phorbol ester and tumor necrosis factor-alpha. Circ Res 90: 143-150, 2002

61. Ikeda RK, Nayar J, Cho JY, Miller M, Rodriguez M, Raz E and Broide DH: Resolution of airway inflammation following ovalbumin inhalation: Comparison of ISS DNA and corticosteroids. Am J Respir Cell Mol Biol 28: 655-663, 2003.

62. Anderson ME: Oxidant stress promotes disease by activating CaMKII. J Mol Cell Cardiol 89: 160-167, 2015.

63. Fujisawa T, Velichko S, Thai P, Hung LY, Huang F and Wu R: Regulation of airway MUC5AC expression by IL-1beta and IL-17A; the NF-kappaB paradigm. J Immunol 183: 6236-6243, 2009.

64. Qu J, Do DC, Zhou Y, Luczak E, Mitzner W, Anderson ME and Gao P: Oxidized CaMKII promotes asthma through the activation of mast cells. JCI Insight 2: e90139, 2017.

65. Zhou Y, Tung HY, Tsai YM, Hsu SC, Chang HW, Kawasaki H, Tseng HC, Plunkett B, Gao P, Hung CH, et al: Aryl hydrocarbon receptor controls murine mast cell homeostasis. Blood 121: 3195-3204, 2013

66. Mahdavinia M, Suh LA, Carter RG, Stevens WW, Norton JE, Kato A, Tan BK, Kern RC, Conley DB, Chandra R, et al: Increased noneosinophilic nasal polyps in chronic rhinosinusitis in US second-generation Asians suggest genetic regulation of eosinophilia. J Allergy Clin Immunol 135: 576-579, 2015.

67. Totlandsdal AI, Cassee FR, Schwarze P, Refsnes M and Låg M Diesel exhaust particles induce CYP1A1 and pro-inflammatory responses via differential pathways in human bronchial epithelial cells. Part Fibre Toxicol 7: 41, 2010.

68. Manners S, Alam R, Schwartz DA and Gorska MM: A mouse model links asthma susceptibility to prenatal exposure to diesel exhaust. J Allergy Clin Immunol 134: 63-72, 2014.

69. Wang H, Do DC, Liu J, Wang B, Qu J, Ke X, Luo X, Tang HM, Tang HL, Hu C, et al: Functional role of kynurenine and aryl hydrocarbon receptor axis in chronic rhinosinusitis with nasal polyps. J Allergy Clin Immunol 141: 586-600.e6, 2018. 